Relations industrielles

Industrial Relations

\title{
Introduction à l'Etude du Travail, Bureau Introduction à l'Etude du Travail, Bureau, 1957, 374 pp.
}

\section{Jean-Paul Deschênes}

Volume 14, numéro 4, octobre 1959

URI : https://id.erudit.org/iderudit/1022148ar

DOI : https://doi.org/10.7202/1022148ar

Aller au sommaire du numéro

Éditeur(s)

Département des relations industrielles de l’Université Laval

ISSN

0034-379X (imprimé)

1703-8138 (numérique)

Découvrir la revue

Citer ce compte rendu

Deschênes, J.-P. (1959). Compte rendu de [Introduction à l'Etude du Travail, Bureau Introduction à l'Etude du Travail, Bureau, 1957, 374 pp.] Relations industrielles / Industrial Relations, 14(4), 626-627.

https://doi.org/10.7202/1022148ar

Tous droits réservés (C Département des relations industrielles de l’Université Laval, 1959
Ce document est protégé par la loi sur le droit d'auteur. L’utilisation des services d'Érudit (y compris la reproduction) est assujettie à sa politique d'utilisation que vous pouvez consulter en ligne.

https://apropos.erudit.org/fr/usagers/politique-dutilisation/ 
ganisation des travailleurs, de l'action législative et de l'action politique. La désintégration progressive qui a abouti à l'événement de 1935 n'était pas pourtant fatale. Même si elle a été conditionnée par les changements industriels et la crise économique, elle n'aurait pas pu se produire si elle ne s'était accompagnée d'une crise de leadership et de laccaparement des postes de commande par les représentants des unions de métiers oubliant qu'une fois à la tête de lAFL ils se devaient de considérer le bien de l'ensemble du groupement.

\& A central conclusion of this monograph, dit l'auteur, is that responsability for the splitting of the labor movement in the 1930's rests primarily with the craft union leaders who controlled the AFL. The writer identifies the AFL national office rather closely with the controlling bloc of craft unions. The national office was in a real sense their organization - it had little authority of its own - and as the leaders of the labor movement the craft unions were, in this writer's view, obliged to see that the movement progressed and that all workers, of whatever skill, had the opportunity to become union members. To the extent that the crafts were indifferent to or were opposed to organizing the unskilled and semiskilled workers they neglected this obligation $\gg$.

Maintenant que l'AFL et le CIO se sont réunis dans une centrale unique, cette question est passée à l'histoire. Il existe bien des travaux la-dessus. Mais l'ouvrage du Dr Morris est sûrement celui qui est le plus à point. En s'appuyant sur une documentation abondante, il présente d'une façon objective et claire tous les aspects de ce problème. C'est une excellente contribution scientifique qui rendra service à tous ceux qui veulent connaître le mouvement ouvrier. Les chefs ouvrier spécialement $y$ trouveront matière à réflexion. Ils pourront de cette histoire tirer par eux-mêmes des leçons utiles qui les éclaireront sur la façon de conoilier des tendances divergentes inévitables dans les groupements qu'ils dirigent.

\section{GÉrard Dion}

Introduction à l'Etude du Travail, Bureau International du Travail, Genève, 1957, $374 \mathrm{pp}$.

Ce volume a le mérite de présenter dans un langage clair et non prétentieux un champ étendu des techniques de la gérance propres à accroître la productivité. De plus, il est un ouvrage de référence très utile à tous ceux qui, de près ou de loin, s'intéressent à l'étude du travail.

L'Introduction d̀ l'Etude du Travail comprend quatre parties. La première s'intitule «Productivité et étude du travail » et examine brièvement le problème général des causes de la faible productivité et de leur élimination par Pemploi de techniques de direction et d'organisation. Cette partie a pour but de situer l'étude du travail parmi les autres moyens dont disposent les chefs d'entreprise pour solutionner des problèmes industriels, et les sujets qu'elle traite sont: Productivité et niveau de vie; La productivité dans l'entreprise; les techniques de direction propres à diminuer le contenu de travail et les temps improductifs; L'étude du travail: Le facteur humain dans l'application de l'étude du travail; et Les conditions de travail.

La seconde partie du volume est consacrée à l'une des techniques de l'étude du travail, soit l'étude des méthodes. Les sujets traités dans cette partie sont: Introduction à l'étude des méthodes et choix des travaux à étudier; Accumulation; Examen et mise au point des données; Circulation et Manutention des matières; Déplacements des travailleurs dans la zone de travail; Méthodes de travail et Mouvements au poste de travail; Mise en application et contrôle de la méthode améliorée.

La troisième partie traite de la mesure du travail, et les sujets étudiés sont: Considérations générales sur la mesure du travail; Etude des temps: matériel; Choix du travail à étudier; Exécution d'une étude des temps; Etude des temps: Jugement d'allure; Examen des données et calcul des temps alloués; Emploi des normes de temps, autres techniques de mesure de travail; Organisation d'un service d'étude du travail.

La quatrième partie contient un certain nombre d'annexes, dont l'une en 
particulier concerne l'utilisation de l'ouvrage comme moyen d'enseignement.

On ne peut que recommander la lecture de cet ouvrage qui présente des méthodes éprouvées et efficaces, qui ne confond pas science technique, et qui ne peut qu'enrichir notre vocabulaire français dans ce champ d'activité.

J.P.D.

La Politique du Patronat français 19361955, Henry W. Ehrmann. Traduit de l'anglais par André Michel. Librairie Armand Colin, 103, Boulevard St-Michel, Paris, 5, 1959, 416 pp. $\$ 3.65$

Cet ouvrage est la traduction française de Organized Business in France que nous avons déjà présenté aux lecteurs de Relations industrielles (vol. 14. no 2 , avril 1959, pp. 302-303). On pourra s'y reporter pour voir ce que nous pensons.

Dans un avant-propos spécial pour l'édition française, l'auteur nous avertit que la traduction présente très peu de différences avec l'édition américaine.
Il a abrégé certaines notes, écourté la discussion de ses sources et condensé certains «portraits 》de personnalités. Ce sont là des changements mineurs.

Nous sommes cependant étonnés des précautions oratoires que prennent l'éditeur et l'auteur pour présenter cet ouvrage au public français. On craint, semble-t-il, que celui-ci n'accepte pas tous les jugements de l'auteur. Comme si cela n'était pas normal dans les études en pareilles matières! Comme si les Français vivaient dans un monde fermé sans avoir connaissance de ce qui se passe ailleurs et que le fait de traduire dans leur langue l'expression d'opinions déjà publiées en changeait la nature! Pour nous du Canada, ces précautions sont inutiles.

Nous avons déjà recommandé cet ouvrage et sommes très heureux que la traduction française le rende accessible à un plus grand nombre de lecteurs dans notre pays. On remarquera aussi qu'il se vend près de la moitié du prix de l'original américain. Et on peut se le procurer aux Presses universitaires Laval, 28 rue Ste-Famille, Québec.

\section{GÉRARD Dion}

\section{PUBLICATIONS RECENTES RECENT PUBLICATIONS}

\section{Généralités}

The Economy of New Jersey: Problems and Prospects. Eleventh Annual LaborManagement Conference, University Commons, New Brunswick, New Jersey, April 21, 1959. Institute of Management and Labor Relations, Rutgers - The State University, 75 pp.

«La paix sociale dans l'entreprise », par L. A. Bekaert, Bulletin Social des Industriels, 71 Ave de Cortenberg, Bruxelles 4, Belgique, no 259, juillet-août 1959, pp. 272-280.

The I.L.O. in a Changing World. Report of the Director-General to the 42 nd Session of the International Labour
Conference, 1958, Twelfth Report of the International Labour Organisation to the United Nations. Report I, International Labour Office, Geneva, 1958, 127 pp.

« Les relations professionnels au Japon », par Isao Kikuchi, Revue Internationale du Travail, Bureau International du Travail, Genève, vol. LXXX, no 2, août 1959, pp. 164-188.

\& Relations humaines, technique ou charité », par R. Pasquasy, Bulletin Social des Industriels, 71 Ave de Cortenberg, Bruxelles 4, Belgique, no 259, juilletaoût 1959 , pp. 288-291. 